\title{
Fitting theories of nuclear binding energies
}

\author{
G.F. Bertsch, B. Sabbey, and M. Uusnäkki \\ Physics Department and Institute for Nuclear Theory, \\ University of Washington, Seattle WA
}

(Dated: November 14, 2018)

\begin{abstract}
In developing theories of nuclear binding energy such as density-functional theory, the effort required to make a fit can be daunting due to the large number of parameters that may be in the theory and the large number of nuclei in the mass table. For theories based on the Skyrme interaction, the effort can be reduced considerably by using the singular value decomposition to reduce the size of the parameter space. We find that the sensitive parameters define a space of dimension four or so, and within this space a linear refit is adequate for a number of Skyrme parameters sets from the literature. We do not find marked differences in the quality of the fit between the SLy4, the BSk4 and SkP parameter sets. The r.m.s. residual error in even-even nuclei is about $1.5 \mathrm{MeV}$, half the value of the liquid drop model. We also discuss an alternative norm for evaluating mass fits, the Chebyshev norm. It focuses attention on the cases with the largest discrepancies between theory and experiment. We show how it works with the liquid drop model and make some applications to models based on Skyrme energy functionals. The Chebyshev norm seems to be more sensitive to new experimental data than the root-mean-square norm. The method also has the advantage that candidate improvements to the theories can be assessed with computations on smaller sets of nuclei.

PACS numbers:
\end{abstract}




\section{INTRODUCTION}

In making theories of nuclear binding energies ("mass formulas"), there are invariably parameters that are determined by fitting the experimental data. If the fitting parameters are not defined properly, they may be underconstrained by the data, causing problems in making the fit. This is the situation for the Skyrme parameterization of the self-consistent mean-field theory (SCMF) of binding energies, also called density-functional theory. While methods for dealing with underconstrained parameters are well known, to our knowledge there has not been a critical analysis of the Skyrme parameterization. We shall show here that the sensitive degrees of freedom in the Skyrme energy functional are very similar to those of the liquid drop model, except for the spin-orbit interaction. When the parameter space is restricted to those degrees of freedom, linear methods can be applied to make a considerable reduction in the effort required for a search. We show how this works in the next section, refitting various parameterizations from the literature.

A second way to reduce the effort in evaluating theories is to use a more economical norm for the fit. Usually one makes a least-squares fit to the binding energies, i.e. using as a norm the root-mean-square (r.m.s.) average of the residual difference $r_{A}$ between theory and experiment,

$$
r_{A}=E_{\text {theory }}(A)-E_{\text {exp }}(A) .
$$

This requires calculating all nuclei. An alternative is the the Chebyshev norm, used in making a minimax fit. It allows one to screen theories and their improvements taking only the information on a small set of nuclei, the "worst cases" of the baseline theory. By calling attention to these cases, it could also be helpful to experimentalists choosing which nuclei to study, and to theorists searching for missing ingredients in the baseline theory. There is an extensive literature on the minimax method [1], but it only rarely used in physics [2]. We describe the method in Sect. III below, applying it to the liquid drop model as a simple exercise and then to SCMF. We also mention that another norm somewhat between the least squares and the C-norm has been proposed for fitting nuclear binding energies [3].

In our discussion below, the Bethe-Weizsäcker semiempirical mass formula will provide a convenient calibration point on the theory. For reference, the formula is given by [4]

$$
B=a_{v} A-a_{s} A^{2 / 3}-a_{c} \frac{Z^{2}}{A^{1 / 3}}-a_{a} \frac{(N-Z)^{2}}{4 A}+\delta \frac{\left((-1)^{N}+(-1)^{Z}\right)}{A^{1 / 2}},
$$


TABLE I: Skyrme energy functional in the $c$-parameterization. The label $i$ is expanded into a integer $n$ and an isospin label $t=0,1$. The isoscalar and isovector densities are $\rho_{0,1}=\rho_{n} \pm \rho_{p}$ and other densities are the same as defined in ref. [5].

\begin{tabular}{c|l}
\hline$i=n t$ & density \\
\hline $1 t$ & $\rho_{t}^{2}$ \\
$2 t$ & $\rho_{t}^{2}\left(\rho_{0}\right)^{\alpha}$ \\
$3 t$ & $\left|\nabla \rho_{t}\right|^{2}$ \\
$4 t$ & $\rho_{t} \tau_{t}$ \\
$5 t$ & $\nabla \rho_{t} \cdot \mathbf{J}_{t}$ \\
\hline
\end{tabular}

where $B$ is the (positive) binding energy of the nucleus $A$.

\section{SKYRME FUNCTIONALS}

The Skyrme energy functional in its present form [5] has 10 linear parameters and one nonlinear parameter, the exponent $\alpha$ of the density-dependent interaction. We shall only fit the linear coefficients, holding $\alpha$ fixed. In practice, the spin-orbit interaction is often assumed to have a specific isospin structure. This reduces the number of linear parameters to nine. Pairing is an indispensable ingredient in the theory but is outside the scope of the Skyrme energy functional. We include pairing following the treatment of ref. [6], using a LipkinNogami treatment of BCS pairing with a surface-enhanced zero-range pairing interaction. The fits are only done on even-even nuclei, so the dependence of the fit on the binding energies would be very much reduced anyway. We represent Skyrme energy functional as in ref. [5] with interaction energy density given by

$$
\mathcal{V}=\sum_{i=1}^{10} c_{i} f_{i}
$$

where $c_{i}$ is a parameter and $f_{i}$ is a function of the one-body densities. The specific forms of the $f_{i}$ needed for time-reversal invariant densities are given in Table I. The interaction energy for each nucleus $A$ requires the integrals over the corresponding densities $f_{i A}$,

$$
I_{i A}=\int d^{3} r f_{i A}
$$


Since binding energy is a nonlinear function of the Skyrme parameters, all of the complications of nonlinear searches are present in the problem of optimizing the functional. However, there are many parameter sets in the literature that we can take as starting points in a search using linear methods. This will determine a local minimum of the chosen norm in the vicinity of the starting parameter set. On a technical level, one needs the derivatives of the binding energies with respect to the parameters to make a linear fit. The required derivatives are easily obtained with the help of the Feynman-Hellman theorem 7] which expresses the derivatives in terms of integrals over the original densities. The theorem is valid for density functional theory because it has a variational character. The linear least squares fit requires inverting the matrix $M$ having elements

$$
M_{i j}=\left(I I^{\dagger}\right)_{i j} \equiv \sum_{A} I_{i A} I_{j A}
$$

The refit parameters are changed by an amount $\Delta c$ given by

$$
\Delta c=M^{-1} I^{\dagger} r
$$

Here $\Delta c$ and $r$ are vectors with components $c_{i}$ and $r_{A}$ respectively.

The main technical problem arises from the redundancy among the parameters. In parameter space, there are flat directions in which the parameter change can be large with only a small effect on the linearized energy. However, if one accepts these large changes, one discovers that they invalidate the linear approximation and the final energies can turn out to be worse than the starting values. This is a common problem in fitting and is typically addressed by a singular value decomposition of the parameter space. In essence, one reduces the dimension of the space to eliminate the flat directions. This is achieved by first diagonalizing the matrix $M$ and projecting onto the subspace spanned by the eigenvectors having eigenvalues larger than some minimum value.

We now show how this works in practice with the SLy4 Skyrme energy functional [8]. The binding energy calculations are performed with the Paris-Brussels code ev8 [9] which requires that occupation probabilities of time-reversed orbitals are equal. Accordingly, we only fit to the even-even nuclei in the mass table. We also restrict the fit to nuclei with $N, Z \geq 8$, as the lightest nuclei are not well described by SCMF. For the SLy4 interaction, the authors of ref. 6] provided us with data on wave functions and equilibrium deformations that we took as a starting point for our refits. 


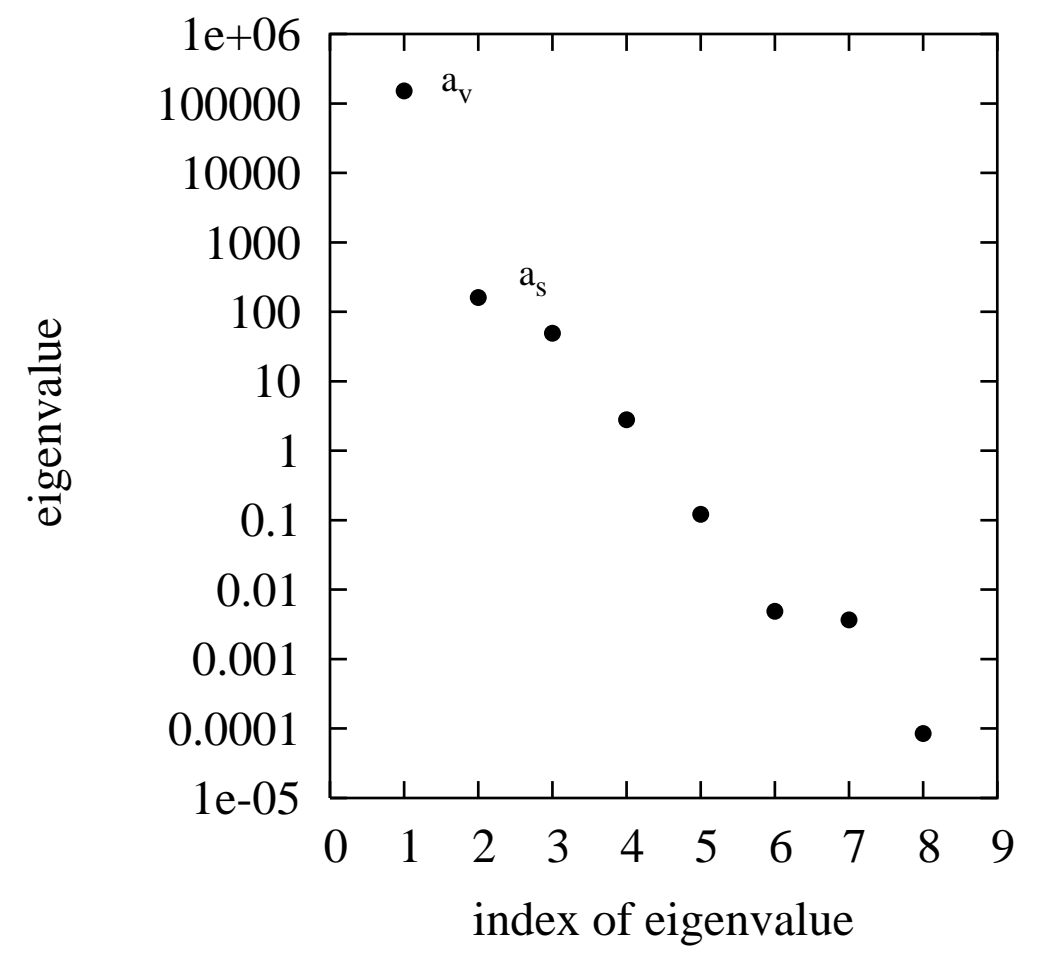

FIG. 1: Eigenvalues of the least square fitting matrix, eq. (2)

The experimental energies are taken from the 2003 mass table [10], which has measured values for 579 even-even nuclei. The SLy4 parameterization was obtained by constraining the parameters by properties other than binding energy. In particular, the equilibrium nuclear matter density $\rho_{0, e q}$ was constrained to a value that yielded a good description of nuclear radii. The condition may be expressed

$$
\frac{d}{d \rho_{0}} \frac{T+V}{\rho_{0}}=\frac{1}{5 m} \frac{k_{f}^{2}}{\rho_{0, e q}}+c_{10} \rho_{0, e q}+(1+\alpha) c_{20} \rho_{0, e q}^{1+\alpha}+c_{40} k_{f}^{2}=0 .
$$

where $k_{f}=\left(3 \pi^{2} \rho_{0, e q} / 2\right)^{1 / 3}$ is the Fermi momentum. We enforce this condition in our fit, leaving 8 independent parameters. We expect that the resulting refit parameterization will maintain a good description of nuclear radii. The eigenvalues for the 8-dimensional matrix $M$ are displayed in Fig. 1, ordered by size. Remarkably, they span a range of nearly 10 orders of magnitude. Also note that the first eigenvalue is 3 orders of magnitude larger than any of the others.

We now consider fits in subspaces of various dimensions $N$, keeping the $N$ most significant 


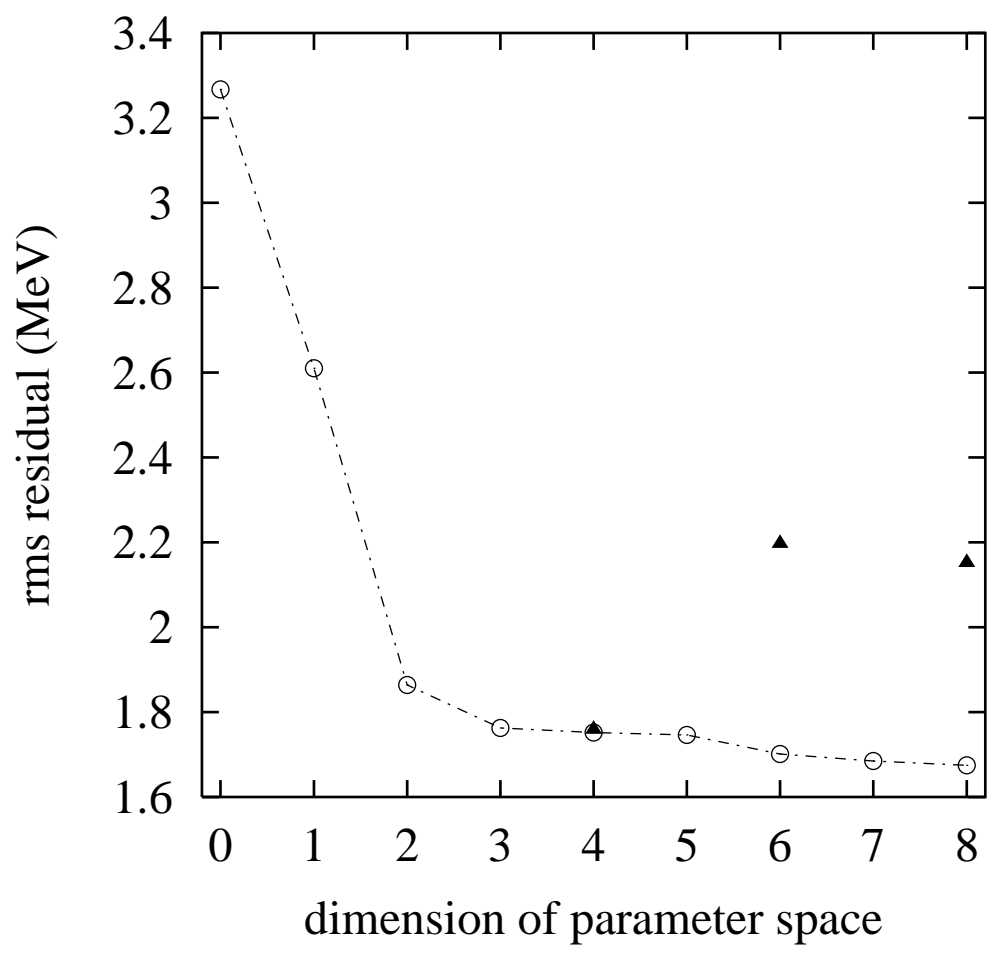

FIG. 2: Least squares refit as a function of the dimension of the parameter space. The r.m.s residuals of the linear refit are shown as circles, connected by lines to guide the eye. The triangles show the actual r.m.s residuals for the parameters determined by the linear refit. However, not all nuclei converged for the $\mathrm{N}=6$ and $\mathrm{N}=8$ parameter sets, and they were not included in the r.m.s. residual.

eigenvectors. The r.m.s. residuals of the linear refits,

$$
\left|r-(\Delta c I)^{\dagger}\right|^{2} \equiv \sum_{A}\left(r_{A}-\sum_{i} \Delta c_{i} I_{i A}\right)^{2}
$$

are shown in Fig. 2 as a function of $N$. The original SLy4 has a $3.3 \mathrm{MeV}$ r.m.s. residual, and this improves to about $1.7 \mathrm{MeV}$ for $N \geq 3$. Thus, it seems that spaces of dimension larger than 3 or 4 are not needed to improve the quality of the fit. However, since this is only a linear refit it must be demonstrated that the quality of the fit is not degraded when one uses the wave functions of the new parameter set. For the cases $N=4,6$ and 8 , we have recalculated the orbitals of all the nuclei to avoid the linear approximation. The iterative procedure to recalculate the orbitals converged as expected for the $N=4$ parameter space, and the new energies were very close to the values obtained by the linear approximation. For the $N=6$ and $N=8$ spaces, there are large changes in some of the parameters and 
the iterative procedure for calculating new orbitals did not always converge. The calculated norms including only converged nuclei are shown as black triangles in Fig. 2. One sees that the norm for the $N=8$ is much larger than predicted by the linear approximation, and even in the $N=6$ case there is significant error. We show in Fig. 3 plots of the residuals as function of $N$ for the SLy4 parameter set and the $N=4$ linear refit. One sees that the refit mainly affects the heavy nuclei, correcting the trend to underbind them. This is at the expense of the region near the doubly magic ${ }^{208} \mathrm{~Pb}$, which is now overbound.

Let us now try to interpret the eigenvectors of $M$. The vector corresponding to the largest eigenvalue is easy to interpret as the direction in parameter space that controls nuclear matter binding energy. To see this, we start with the expression for the nuclear matter binding energy in term of the $c$ parameters,

$$
a_{v}(c)=\frac{3 k_{f}^{2}}{10 m}+c_{10} \rho_{0, e q}+c_{20} \rho_{0, e q}^{1+\alpha}+\frac{3}{5} c_{40} k_{f}^{2} \rho_{0, e q} .
$$

The direction vector with the maximum sensitivity to $a_{v}(c)$ is proportional to the gradient $\vec{\nabla}_{c} a_{v}(c)$. We find that the overlap of this direction vector with the first eigenvector of $M$ is $99 \%$. Thus, the extremely sensitive direction revealed by the eigenvalue plot Fig. 2 is nothing more than the nuclear matter binding energy. Its new value in the refit is -16.06 $\mathrm{MeV}$.

Another important property of nuclear matter is the symmetry energy, given by

$$
a_{s}(c)=\frac{k_{f}^{2}}{6 m}+c_{11} \rho_{0, e q}+c_{21} \rho_{0, e q}^{1+\alpha}+\left(c_{41}+\frac{c_{40}}{3}\right) k_{f}^{2} \rho_{0, e q}
$$

It does not correspond quite so well to a particular eigenvector: the direction vector along $\vec{\nabla}_{c} a_{s}(c)$ has a $53 \%$ overlap with the second and an $80 \%$ overlap with the third eigenvector. However, within the space of the first three eigenvectors, a vector with an overlap of $98 \%$ can be constructed. We can therefore say that the $N=3$ space includes parameters for both the nuclear matter binding energy and the symmetry energy. The three other adjustable parameters in the liquid drop model are associated with the surface energy, the Coulomb energy, and the pairing energy. It is likely, but we have not checked, that the surface energy of semi-infinite nuclear matter provides a third vector for spanning the $N=3$ space of the first three eigenvectors of $M$. The remaining liquid drop parameters are not relevant to our fits, since the Coulomb interaction and the pairing are not adjusted. The fourth eigenvector of $M$ contains physics that is entirely missing from the liquid drop model, namely the spin-orbit 


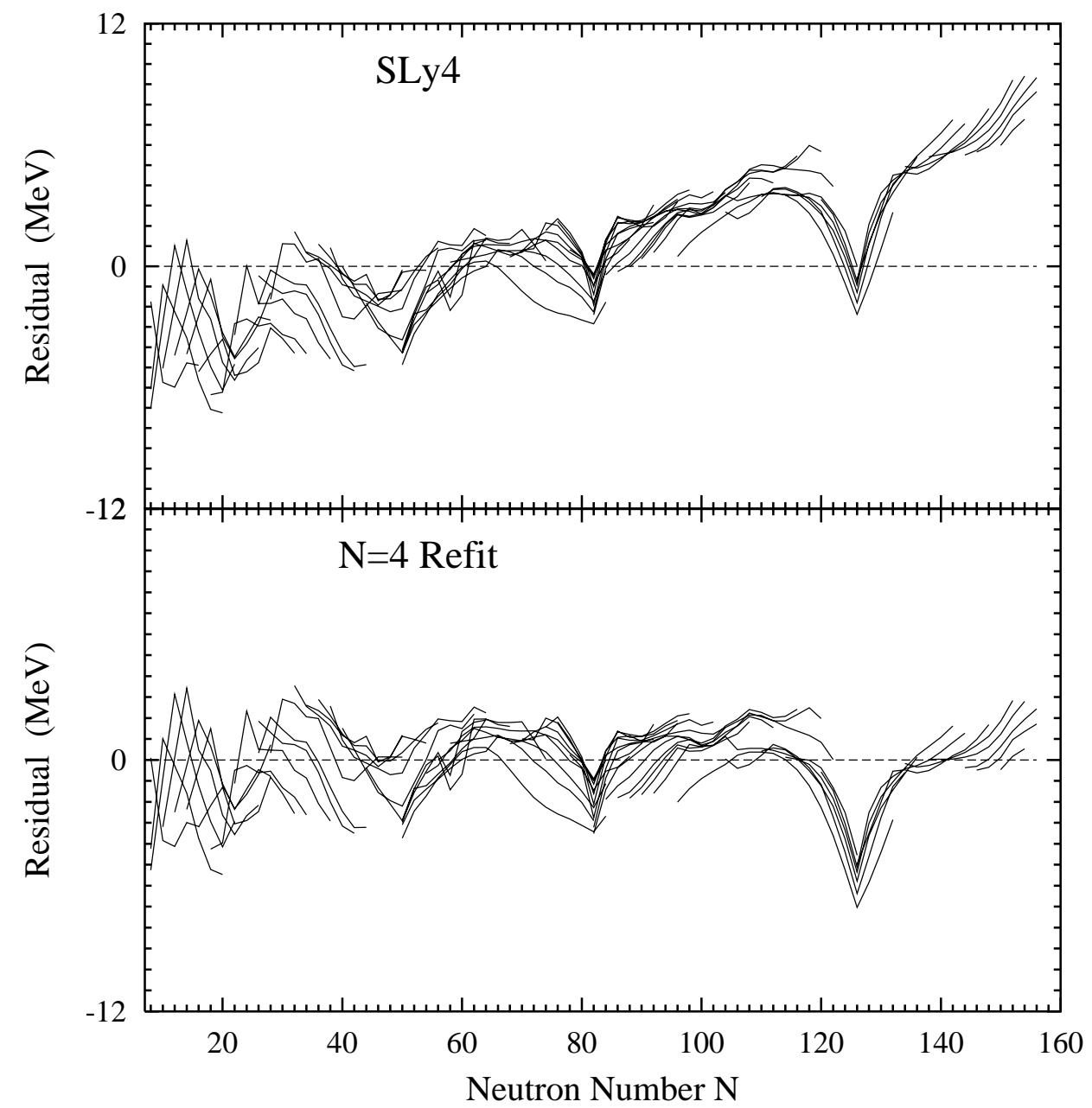

FIG. 3: Residuals between theory and experiment as a function of $N$, with nuclei of the same atomic number joined by lines. The top plot shows the original SLy 4 and the $N=4$ linear refit is shown on the bottom. 
TABLE II: Refits of various Skyrme parameterizations. The experimental data set is even-even nuclei of the 2003 mass table [10]. The last entry give the corresponding properties of the liquid drop model, eq. (1), for comparison purposes. All energies are in $\mathrm{MeV}$.

\begin{tabular}{|c|lll|}
\hline Theory & $\begin{array}{l}\text { r.m.s } \\
\text { residual }\end{array}$ & & \\
\hline SLy4 [8] & 1.75 & -16.06 & 32.0 \\
\hline SkP-based [11] & 1.75 & -16.11 & 31.1 \\
\hline BSk4-based [12] & 1.65 & -16.03 & 29.6 \\
\hline Skxce-based [13] & 1.55 & -16.10 & 31.0 \\
\hline LD & 3.1 & -15.6 & 23.3 \\
\hline
\end{tabular}

interaction. In fact, there is a 90\% overlap between that vector and the relevant Skyrme parameter, $c_{5 t}$ in the notation of Table I. Beyond the spin-orbit interaction, the Skyrme parameters do not provide additional sensitive determinants of the binding energies.

There may be multiple local minima in the parameter space of the Skyrme functional, and other parameterizations in the literature may reside in other minima. It is therefore of interest to see what the refitting procedure produces for them. We have carried this out for the $\mathrm{SkP}$ parameterization of ref. [11], the BSky4 of ref. [12] and the Skxce of ref. [13]. However, there is an important caveat in interpreting the results. The quoted parameterizations were generated taking different approximations for various non-Skyrme energy terms, while our calculations here only vary the Skyrme parameters themselves, keeping the same treatment of the other terms the same as in the SLy4 calculation. Thus, our extracted r.m.s. residuals will not be directly comparable to the quoted residuals from the original fits. In Sect. IV below, we will explicitly examine the effect of some of these ancillary approximations on the fit. Another caveat is that we start from the same deformations as with SLy4. The deformation is allowed to change in the solution of the mean-field equations, but there could be a lower energy state in some other well of the deformation energy landscape.

Having obtained the wave functions for the different parameterizations, we extract the density integrals and eigenvectors and then apply the $N=4$ linear refit. The results are shown in Table II. The main eigenvectors of the SkP and the BSky4 were very similar to that 
of SLy4, and the linear refits did not need a substantial adjustment of the parameters. The $\mathrm{SkP}$ has the same density dependence $(\alpha=1 / 6)$ as the SLy4, and in fact we see from the table that the quality of the fit is virtually identical. The BSky4 has a density dependence $\alpha=1 / 3$, the value that is found for the many-body theory of a dilute Fermi gas. Finally, we have also considered a parameterization with a density dependence $\alpha=1 / 2$, the Skxce of

ref. [13]. In this case, the original parameter set did not give an acceptable fit for applying the linear refit. We therefore made some iterations on the fit to get a good starting point.

Comparing the different parameter sets, we see that there is very little difference between the qualities of the fits, all of them being in the range of $1.5-1.7 \mathrm{MeV}$. It is interesting to calibrate this number by comparing with the r.m.s. residual of the liquid drop model. The result of fitting eq. (1) to the 2003 nuclear mass table [10] gives an r.m.s. residual of 2.95 $\mathrm{MeV}$; we quote in the last line of Table II the fit to even-even nuclei only. We see that the SCMF achieves a factor of two improvement in the calculated binding energies. Of course the SCMF has twice as many parameters, but as we just saw that many are superfluous from the point of view of the binding energies. Still, one might have hoped for a more dramatic improvement given the computational cost of the SCMF as compared to the liquid drop formula.

\section{MINIMAX FITS}

We now consider a completely different fitting criterion, the Chebyshev norm. The Chebyshev norm $\epsilon$ is defined as the maximum absolute value of the residuals $r_{A}=E_{\text {data }}-E_{\text {theory }}$,

$$
\epsilon=\max _{A}\left|r_{A}\right|,
$$

We shall call this value the "C-norm" for short. The object of the fit is of course to minimize $\epsilon$, hence the designation "minimax fit". In general, if the theory has $N$ adjustable parameters, there will be $N+1$ members of the set that have a residual equal to $\epsilon$. We call these the critical cases. In searching for a better theory, one can screen candidates by just testing them on this set. If the new theory does not produce a smaller $\epsilon$ on the critical set, it can be immediately rejected.

We perform the minimax fit using the Chebyshev norm as follows. For an $N$-parameter theory, one first selects a set of $N+1$ nuclei and makes the fit with them. This can be 
TABLE III: Liquid drop model, comparing least squares fits with minimax fits of the 2003 and 1995 mass tables 10$]$. The fit does not include light nuclei $(N$ or $Z<8)$.

\begin{tabular}{|c|c|c|c|}
\hline Data set & $\begin{array}{l}\text { r.m.s. } \\
(\mathrm{MeV})\end{array}$ & $\begin{array}{l}\text { C-norm } \\
(\mathrm{MeV})\end{array}$ & $\begin{array}{l}\text { overbound critical nuclei } \\
\text { underbound critical nuclei }\end{array}$ \\
\hline 2003 & 2.9 & 9.2 & $\begin{array}{l}{ }^{40} \mathrm{Ar},{ }^{76} \mathrm{Se},{ }^{77} \mathrm{Br},{ }^{229} \mathrm{Fr} \\
{ }^{100} \mathrm{Sn},{ }^{132} \mathrm{Sn}\end{array}$ \\
\hline 1995 & 3.0 & 8.0 & $\begin{array}{l}{ }^{73} \mathrm{Ge},{ }^{101} \mathrm{Nb},{ }^{230} \mathrm{Ra} \\
{ }^{23} \mathrm{O},{ }^{132} \mathrm{Sn},{ }^{207} \mathrm{~Pb}\end{array}$ \\
\hline $\begin{array}{c}2003 \\
\sigma<0.2 \mathrm{Mev}\end{array}$ & 2.8 & 8.4 & $\begin{array}{l}{ }^{40} \mathrm{Ar},{ }^{73} \mathrm{As},{ }^{76} \mathrm{Se},{ }^{229} \mathrm{Fr} \\
{ }^{102} \mathrm{Sn},{ }^{132} \mathrm{Sn}\end{array}$ \\
\hline
\end{tabular}

done by the least squares method, which yields equal residuals for $N+1$ nuclei. Then the set is updated by replacing members with other nuclei until a set is found that satisfies the minimax condition. It is easy to choose a nucleus to add to the set-simply take the nucleus with the largest residual. It is not obvious which nucleus should be replaced. The ascent algorithm described in ref. 1] gives a procedure which we found to be quite robust, usually coming to the critical set after less than ten iterations.

We first apply the minimax fit to the 5-parameter liquid drop model, eq. (11). Results are given in Table III. For the first row, the formula was fitted to the 2149 nuclei in the 2003 mass table having $N, Z \geq 8$. Fitting to optimize the C-norm gives $\epsilon=9.2 \mathrm{MeV}$, while fitting by least squares gives an r.m.s. residual of $2.9 \mathrm{MeV}$ [14].

The 6 critical nuclei of the minimax fit are given in the fourth column of the table and their positions on the chart of nuclides is shown in Fig. 4. We see that the critical nuclei are spread out over the entire mass range. Two of the nuclei, ${ }^{229} \mathrm{Fr}$ and ${ }^{132} \mathrm{Sn}$, are at the neutron-rich border of the mass table, and two are doubly magic: ${ }^{100} \mathrm{Sn}$ and ${ }^{132} \mathrm{Sn}$. One might expect that the $N=Z$ line is problematic and indeed ${ }^{100} \mathrm{Sn}$ is on the line and ${ }^{40} \mathrm{Ar}$ is near it. The remaining nuclei are ${ }^{76} \mathrm{Se}$ and ${ }^{77} \mathrm{Br}$ which do not have any particular properties that we are aware of.

It is interesting to compare with the fit to the 1995 mass table. This is shown on the second line in the table. The r.m.s. residual is actually lowered by the added nuclei in the 2003 table, but the C-norm is increased by more than an MeV. This suggests that the 


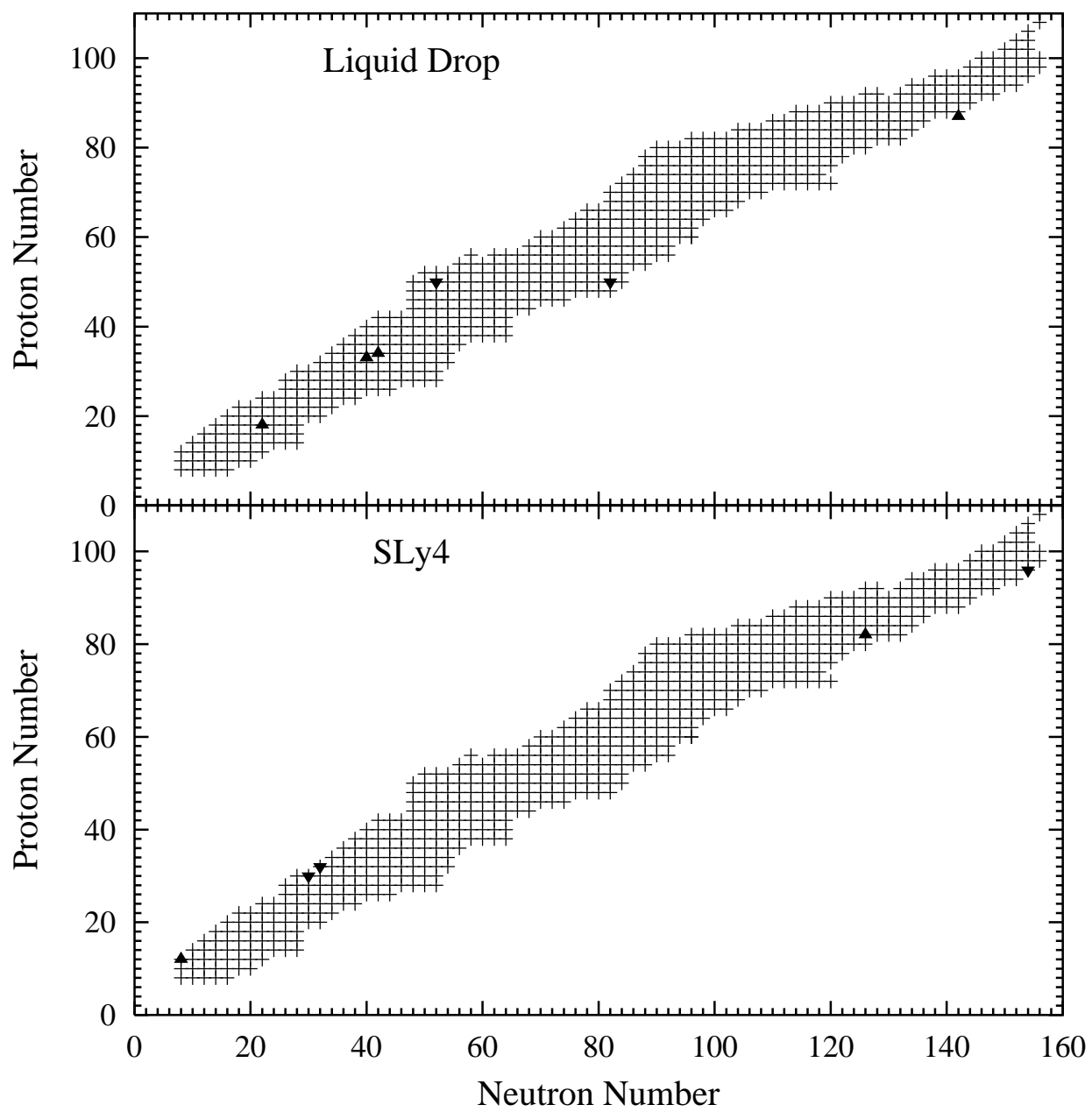

FIG. 4: Chart of nuclei showing the critical nuclei of the liquid drop model and of the linearized refit of the SCMF based on the SLy4 interaction. Critical nuclei are indicated by triangles, with the orientation of the triangle distinguishing overbound $(\Delta)$ and underbound $(\nabla)$ nuclei. The cross area shows the nuclei whose masses have been measured. 
C-norm provides a better discrimination power to exhibit the increased demands imposed by additional data. Most of the added nuclei were evidently easy ones for the formula, decreasing the least squares norm. It is instructive to see which nuclei caused the changes to the minimax fit. Several of the critical nuclei are different in the two fits, but only one of these nuclei is new to the 2003 mass table, namely ${ }^{100} \mathrm{Sn}$. In fact this nucleus is not well determined experimentally; the error in its quoted experimental mass is $0.7 \mathrm{MeV}$, larger than the intended accuracy of the theory. Restricting the 2003 mass table to nuclei having errors less than $200 \mathrm{keV}$, the C-norm decreases by $0.8 \mathrm{MeV}$. This is shown on the third line of the Table. One sees that the nucleus ${ }^{100} \mathrm{Sn}$ is replaced by ${ }^{102} \mathrm{Sn}$ and the $\mathrm{C}$-norm now is not so much greater than in 1995 mass table. Thus, the minimax fitting process identifies ${ }^{100} \mathrm{Sn}$ as an important nucleus to measure more accurately, and it also provides justification for the concentration of theoretical effort on the Sn isotope chain.

We next discuss minimax fits of the Skyrme energy functional. Table IV shows the results of the $N=4$ refits to the Skyrme energy functionals discussed earlier. For SLy4, shown on the top line, the five critical nuclei include two on the $N=Z$ line $\left({ }^{60} \mathrm{Zn}\right.$ and $\left.{ }^{64} \mathrm{Ge}\right)$, one on the very proton-rich side $\left({ }^{20} \mathrm{Mg}\right)$, and two heavy nuclei on the neutron-rich side. One of them $\left({ }^{208} \mathrm{~Pb}\right)$ is doubly magic and another is close to the heaviest known $\left({ }^{254} \mathrm{Cf}\right)$. Overall, the nuclei are well spread as a function of mass number $A$. The SkP functional is similar to SLy4 in its density dependence and isospin dependence of the spin-orbit interaction. As may be seen in the Table, the C-norm of our SkP-based functional is nearly identical to that of SLy4, but the set of critical nuclei is quite different. The set includes a nucleus on the $N=Z$ line, but the mass number is four units lower than the critical nucleus ${ }^{60} \mathrm{Zn}$ of SLy4. The set lacks a doubly magic nucleus, and the only magic number appearing is the $Z=82$ of the critical nucleus ${ }^{214} \mathrm{~Pb}$. The other underbound nuclei are ${ }^{190} \mathrm{~W}$ and ${ }^{192} \mathrm{Os}$, both of which are deformed and on the border between prolate and oblate shapes. These results shown that the specific nuclei on the critical list can change easily from one functional to another. However, the value of the C-norm is quite robust under small changes in the starting parameters.

We next consider a Skyrme parameterization based on the BSk4 functional of Goriely, et al. 12]. Here the the density dependence is $\alpha=1 / 3$ rather than $1 / 6$. As mentioned earlier, our results will not be directly comparable to theirs because of different treatments of the pairing interaction and also additional terms that they have added for their published fits. 
TABLE IV: Minimax refits of Skyrme parameterizations, starting from parameter sets from the literature, and experimental data from the 2003 mass table, including only energies with errors less than $200 \mathrm{keV}$. The critical nuclei are identified by proton number $Z$ and neutron number $N$ as $(Z, N)$. The corresponding fit with the liquid drop model is shown on the last line.

\begin{tabular}{|c|c|c|c|}
\hline Theory & \multirow{2}{*}{$\begin{array}{c}\text { C-norm } \\
\text { (MeV) }\end{array}$} & \multicolumn{2}{|c|}{ critical nuclei } \\
\cline { 3 - 4 } & 4.8 & $(12,8),(82,126)$ & $(30,30),(32,32),(96,154)$ \\
\hline SLy4 [8] & 4.8 & $(42,58),(82,132)$ & $(28,28),(74,116),(76,116)$ \\
\hline SkP-based [11] & 4.8 underbound \\
\hline BSk4-based [12] & 4.7 & $(12,8),(50,52),(82,128)$ & $(38,38),(38,62)$ \\
\hline Skxce-based [13] & 4.4 & $(12,8),(92,126)$ & $(34,34),(38,38),(38,64)$ \\
\hline LD & 8.1 & $(50,52),(50,132),(82,126)$ & $(34,42),(88,142)$ \\
\hline
\end{tabular}

Also, we have taken the deformations from the SLy4 results, which could introduce some error. The results displayed on the third line in Table V show a slight improvement of both norms with respect to the SLy4 interaction. The critical nuclei are different except for ${ }^{20} \mathrm{Mg}$ and ${ }^{208} \mathrm{~Pb}$. There is also an $N=Z$ nuclei in this critical set, ${ }^{76} \mathrm{Sr}$. In fact, the authors of ref. 12] introduce a phenomenological term in the binding energies just to improve the fit near $N=Z$.

The last functional we consider is based on the Skxce parameterization having a density dependence $\alpha=1 / 2$ and a different isospin dependence of the spin-orbit interaction. As seen in Table $\mathrm{V}$, it yields a somewhat better C-norm than the $\alpha=1 / 6$ parameterizations, which is in line with its better performance on the least squares norm. Most of the critical nuclei are different from the previous cases, with only ${ }^{20} \mathrm{Mg}$ having appeared earlier. Like the SLy4, there are two midmass nuclei on the $N=Z$ line, but their identities are different. The only appearance of a spin-orbit shell closure in this set is the $N=126$ neutron shell of the nucleus ${ }^{218} \mathrm{U}$. Also, quite different from the other fits, the most neutron-rich representative is a midmass nucleus. 


\section{TESTING THEORY EXTENSIONS}

There are two ways one can try to improve the theory. One is to introduce new theoretical ingredients or different approximations keeping the same set of parameters to be fitted, and the other is to add terms with additional parameters. In this section we consider some examples of the first approach, examining the effects on the C-norm.

For our first example, we ask how the treatment of the correlation energy associated with center-of-mass motion affects the fit. In principle SCMF is lacking that energy because the state it describes is localized in space. However, all the calculations done with the code ev8 include a one-body contribution to the center-of-mass energy calculated by replacing the nucleon mass by its reduced mass. A better approximation requires calculating the twobody contributions to the center-of-mass energy; an approximation formula for the total is given in ref. [13] as

$$
E_{c m}=-\frac{3}{4}\left(\frac{45}{A^{1 / 3}}-\frac{25}{A^{2 / 3}}\right)
$$

In Table $\mathrm{V}$ we apply that correction to the five nuclei of the SLy4 critical set, first taking out the one-body contribution and then adding $E_{c m}$ from eq. (3) to the energies. One sees only a tiny change in the resulting $\mathrm{C}$-norm. This shows that it is not worthwhile to put a lot of effort in making a better treatment of that term, when the goal is a global improvement of the calculated binding energies. Of course, this example was so simple that the correction could have been easy applied to the mass table as a whole, but it illustrates the point that one may be able to assess the effect of the correction with a much smaller set.

The next example we consider has to do with the treatment the Coulomb energy. In the SCMF calculations reported above, the direct part of Coulomb energy was calculated by solving the Laplace equation for the Coulomb potential using the SCMF charge density. The Coulomb exchange energy was calculated in the local density approximation, as in eq. (3) of ref. [13]. This underestimates the actual displacement energies of isospin partners, as is well known as the Nolen-Schiffer anomaly. We in fact see evidence of that in the BSk4 critical set which has a member ${ }^{40} \mathrm{Ti}$, an isospin partner of the stable nucleus ${ }^{40} \mathrm{Ar}$. In fact the latter nucleus is critical for the liquid drop model. The experimental energy difference between these two nuclei is $29.3 \mathrm{MeV}$. The theoretical difference in the SLy4 theory is 27.5 $\mathrm{MeV}$ including the exchange, but 29.2 without exchange. This suggests eliminating the exchange term in doing the refit. Again, we try it on the critical nuclei first. The results 
are shown on the third line of Table IV. Here the fit is actually worse. In this case we can identify the reason. The baseline theory has a serious problem in the overbinding of the doubly magic nucleus ${ }^{208} \mathrm{~Pb}$. In the fit, the heavier nonmagic nucleus ${ }^{254} \mathrm{Cf}$ becomes critical with the opposite sign on the residual. The parameter variation in the fits hardly affects this difference, so any change in the theory that increases it will be difficult to compensate for. This is the case for the Coulomb exchange, which is larger for ${ }^{254} \mathrm{Cf}$ than for ${ }^{208} \mathrm{~Pb}$.

The last example is inspired by the study of Bender, et al., 6] on the correlation energy. A global table of correlation energies was calculated in that work, with the intention of adding them to the Skyrme energies. Of course the Skyrme parameters must be refitted when the correlation energies are added. We can take their numbers for the critical nuclei to assess the quality of the improvement (if any). We consider as a candidate improvement the simplest version of the correlation energy, the effect of projection on angular momentum zero. These projected energies are calculated with the promesse code [15]. The difference in energies from the two codes is taken as an additive correction, and a refit is performed on the critical nuclei. The results of this exercise is shown on the fourth line of Table IV. In this case the C-norm is decreased by $10 \%$. This gives one some encouragement to carry out the program of ref. [6], applying the projections to the mass table as a whole. Of course, it might still turn out that other nuclei might become worse in the fit with no gain in the C-norm, but the correction at least passes a preliminary screen. The code promesse also projects states of fixed particle numbers $N, Z$ from the BCS wave function. The effect of the particle number projection can be isolated by turning it off in the promesse code, and using the difference as a correction factor. The result, shown on the last line, is another 10 $\%$ improvement in the C-norm. We have also checked that the improvements are additive taking the $J=0$ projection and the $N, Z$ particle number projections together.

\section{FINAL REMARKS}

Fitting nuclear binding energies with theories based on quantum many-body theory is a challenging task, partly due to the large data set that needs to be computed. We believe that work in developing such theories can be reduced by using the Chebyshev norm as we have illustrated. To encourage this effort, our computer program that carries out the minimax fits is available on the Web [16]. 
TABLE V: Testing possible improvements of theory, starting from the SCMF with the SLy4 interaction. The putative improvement is calculated on the SLy4 critical nuclei shown in Table IV.

\begin{tabular}{|c|c|}
\hline Theory & $\begin{array}{c}\text { C-norm } \\
(\mathrm{MeV})\end{array}$ \\
\hline Baseline SLy4 & 4.84 \\
\hline c.m. correlation & 4.69 \\
\hline no Coulomb exchange & 4.89 \\
\hline$J=0$ projection & 4.36 \\
\hline$N, Z$ projection & 4.40 \\
\hline
\end{tabular}

An issue still remains of which set of critical nuclei is best for testing new theoretical ideas. Unfortunately, each Skyrme parameterization points to a different set. We believe that there are clusters of nuclei that come out close to the C-norm limit, and that it shouldn't be so significant which set is used, as long as all the nuclei of some critical set are included. For definiteness, we propose using the SLy4 set of five nuclei, since it has a variety of types with a clear example of a doubly magic nucleus.

For either the r.m.s. or the C-norm, the results of our refitting answers a basic question about the accuracy of SCMF as applied to nuclear binding energies. Namely, how does the quality of SCMF fits including single-particle quantum mechanics compare with the liquid drop model which has no quantum mechanics at all? Comparing on the even-even nuclei in the 2003 mass table, we found that the SCMF can double the accuracy for the r.m.s. norm and do somewhat better for the C-norm. The nominal number of Skyrme parameters is more than twice the number of liquid drop parameters, but in fact all but about four could have been chosen from other considerations. It is of course reassuring that the SCMF is a more predictive theory, and now the challenge is find good ways to extend it.

\section{Acknowledgments}

We thank H. Flocard, R. Furnstahl, D. Lunney and G. Audi for conversations and P.H. Heenen and M. Bender for helpful advice and for making their codes available to us. We also thank T. Duguet for a careful reading of the manuscript which led to some changes and 
clarifications. This work was supported by the U.S. Department of Energy, Office of Nuclear Physics, under Contract DE-FG02-00-ER41132.

[1] E.W. Cheney, "Approximation theory," (American Mathematical Society, Providence, 1982), p. $28-56$.

[2] F. James, Nucl. Inst. Meth. Phys. Res. 211145 (1983).

[3] C. Borcea and G. Audi, "New methods for extrapolating masse far from stability", http://csnwww.in2p3.fr/AMDC/extrapolations/bernex.pdf

[4] B. Povh, K. Rith, C. Scholz and F. Zetsche, "Particles and Nuclei," (Springer, Heidelberg, 1995), p. 19.

[5] M. Bender, P.-H. Heenen, and P.-G. Reinhard, Rev. Mod. Phys. 75, 121 (2003).

[6] M. Bender, G.F. Bertsch, and P.-H. Heenen, nucl-th/0410023.

[7] R.P. Feynman, Phys. Rev. 56340 (1939); H. Hellmann, Einfuehrung in die Quantenchemie (Deuticke, Leipzig, 1937).

[8] E. Chabanat, P. Bonche, P. Haensel, J. Meyer, and R. Schaeffer, Nucl. Phys. A635, 231 (1998), Nucl. Phys. A643, 441(E) (1998).

[9] P. Bonche, et al., Nucl. Phys. A443 39 (1985).

[10] G. Audi, A. H. Wapstra, and C. Thibault, Nucl. Phys. A729, 337 (2003); the data file is available at http://www.nndc.bnl.gov/amdc/masstables/ Ame2003/mass . mas03.

[11] J. Dobaczewski, et al., Nucl. Phys. A422 103 (1984).

[12] S. Goriely, et al., Phys. Rev. C 68054325 (2003).

[13] B.A. Brown, Phys. Rev. C 58220 (1998).

[14] The parameters of the two fits are significantly different; the C-norm of the r.m.s. fit is 50\% higher than in the minimax fit and the r.m.s. of the residuals of the minimax fit is $60 \%$ larger than in the least squares fit.

[15] M. Bender and P.-H. Heenen, Nucl. Phys. A713 390 (2003); A. Valor, P.-H. Heenen, and P. Bonche, Nucl. Phys. A671 145 (2000).

[16] The computer program cheby ·py may be downloaded from

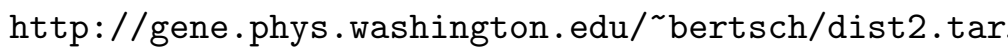

